\title{
Photovoltaic Effect and Space Charge Limited Current Analysis in $\mathrm{TlGaTe}_{2}$ Crystals
}

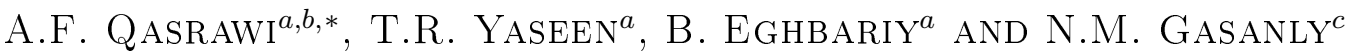 \\ ${ }^{a}$ Department of Physics, Arab-American University, Jenin, West Bank, Palestine \\ ${ }^{b}$ Group of Physics, Faculty of Engineering, Atilim University, 06836 Ankara, Turkey \\ ${ }^{c}$ Department of Physics, Middle East Technical University, 06531 Ankara, Turkey
}

(Received December 8, 2010)

\begin{abstract}
Anisotropic space charge limited current density analysis and photovoltaic effect in $\mathrm{TlGaTe}_{2}$ single crystals has been investigated. It is shown that, above $330 \mathrm{~K}$, the crystal exhibits intrinsic and extrinsic type of conductivity along ( $c$-axis) and perpendicular ( $a$-axis) to the crystal's axis, respectively. The current density $(J)$ is found to be space charge limited. It is proportional to the square and three halves power of voltage $(V)$ along the $a$ - and $c$-axis, respectively. Along the $a$-axis and at sufficiently low electric field values, the activation energy of the current density is found to depend on the one half power of electric field. At high electric fields, the activation energy is field invariant. This behavior is found to be due to the Pool-Frenkel effect and due to a trap set located at $0.26 \mathrm{eV}$, respectively. Along the $c$-axis the crystal is observed to operate under the Child-Langmuir space charge limited regime. $\mathrm{TlGaTe}_{2}$ crystals are found to exhibit photovoltaic properties. The open circuit photovoltage is recorded as a function of illumination intensity at room temperature.
\end{abstract}

PACS: $72.20 .-\mathrm{i}, 72.20 . \mathrm{Ht}, 72.40 .+\mathrm{w}$

\section{Introduction}

In low-dimensional materials electronic transitions, like the charge-density-wave transition, are often observed. Namely, the one-dimensionality is presented by many features which qualitatively differ from those at higher dimensionalities. It has been reported that a one-dimensional (1D) system cannot present a thermodynamic transition at finite temperature owing to the very large fluctuations associated with the $1 \mathrm{D}$ character [1]. In practice, the one-dimensional electronic transitions are observed suggesting that transverse interactions play a significant role. However, in systems where these interactions are weak, the main characteristic features of one-dimensionality remain distinguishable.

$\mathrm{TlInSe}_{2}, \quad \mathrm{TlInTe}_{2}$, and $\mathrm{TlGaTe}_{2}$ are quasi-one-dimensional solids group which construct the chains parallel to the $\boldsymbol{c}$-axis. These characters limit the crystal's physical properties. Some of the reported interesting features [2-13] of these crystals are switching phenomena and negative-differential resistance effects $[5,6]$ which allow $\mathrm{TlGaTe}_{2}$ crystal to play an important role in technological applications such as memory devices. Such properties make the $\mathrm{TlGaTe}_{2}$ crystal attractive for researchers. As for example, the thermal expansion and isothermal compressibility [7], the band structure and permittivity [13] of the crystal have been studied.

In our previous works we have reported the Hall effect analysis [9] and the photoconductivity and recombination

* corresponding author; e-mail: aqasrawi@atilim.edu.tr kinetics [10] in $\mathrm{TlGaTe}_{2}$ crystals. The Hall effect analysis revealed a carrier effective mass, an acceptor and donor concentrations of $0.73 \mathrm{~m}_{0}, 4.10 \times 10^{17} \mathrm{~cm}^{-3}$ and $1.20 \times 10^{17} \mathrm{~cm}^{-3}$, respectively. It was shown that the Hall mobility of $\mathrm{TlGaTe}_{2}$ is limited by the scattering of hole-acoustic phonon interactions. The photoconductivity analysis allowed the determination of recombination center energy as $110 \mathrm{meV}$. The main purpose of this work is to study and discuss the space charge effects on the current transport mechanism along and perpendicular to the crystals axis. Some of the photovoltaic properties of compound $\mathrm{TlGaTe}_{2}$ will also be reported.

\section{Experimental details}

$\mathrm{TlGaTe}_{2}$ polycrystals were synthesized from the high purity elements (at least $99.999 \%$ ) taken in stoichiometric proportions. Single crystals of $\mathrm{TlGaTe}_{2}$ were grown by the Bridgman method in evacuated $\left(10^{-5}\right.$ Torr $)$ silica tubes with a tip at the bottom. The ampoule was moved in a vertical furnace through a thermal gradient of $30^{\circ} \mathrm{C} / \mathrm{cm}$, between the temperatures 775 and $425^{\circ} \mathrm{C}$ at a rate of $1.0 \mathrm{~mm} / \mathrm{h}$. The resulting ingots (grey in color) showed good optical quality and the cleaved mirror-like surfaces contained the chains parallel to the crystal $c$-axis extending along the [001] direction. The X-ray diffraction patterns show that these crystals have tetragonal structure with the lattice parameters: $a=0.8432$ and $c=0.6863 \mathrm{~nm}$. The resulting single crystals were not subjected to any additional annealing. Typical dimensions of the Hall bar-type samples were $15 \times 3 \times 0.2 \mathrm{~mm}^{3}$. For reliable electrical measurements, the electrical con- 
tacts were made by painting high-purity silver paste using suitable masks. The ohmic nature of the contacts was confirmed by the $I-V$ characteristics, which is found to be linear and independent of the reversal current for low applied voltages. The illumination was done using a halogen lamp. The light intensity was altered by changing the distance and/or the value of the current through the lamp and was calibrated using an IL 1700 radiometer.

\section{Results and discussion}

In addition to the process of ohmic electrical conductivity in the $\mathrm{TlGaTe}_{2}$ crystal arising from the presence of thermally excited free carriers, the crystal is able to support relatively large currents resulting from the process of direct current injection from suitable electrodes - the so-called space charge limited (SCL) current.

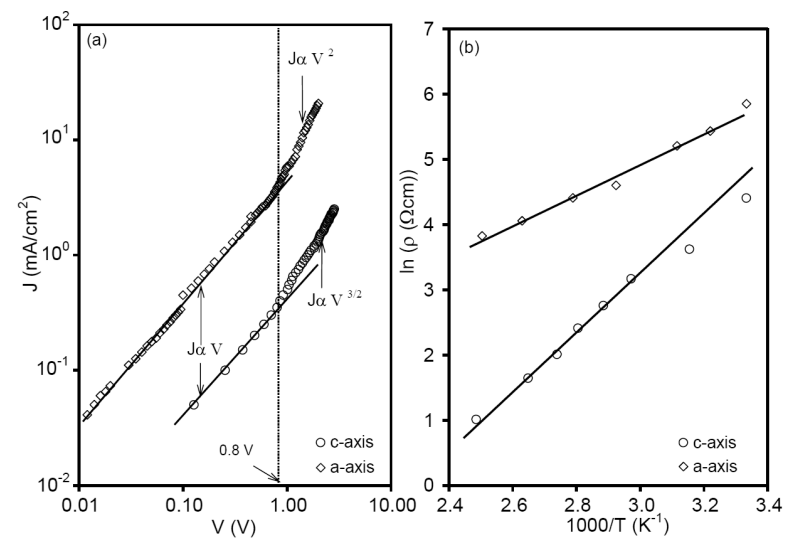

Fig. 1. (a) The $J-V$ plots for $\mathrm{TlGaTe}_{2}$ at $300 \mathrm{~K}$. (b) The $\rho-T^{-1}$ dependence between 300 and $400 \mathrm{~K}$.

Figure 1a illustrates the typical room temperature dark current density-voltage $(J-V)$ characteristic for $\mathrm{TlGaTe}_{2}$ crystals being recorded along the $a$ - and $c$-axis of the crystal. For both measurements, the figure reveals the existence of two distinct regions of $J-V$ curves. At low voltages, $J-V$ characteristic curves possess an ohmic behavior, where the concentration of injected carriers is less than the concentration of free carriers under equilibrium conditions. For voltages greater than $0.8 \mathrm{~V}$, the current density becomes proportional to the square of the voltage along the $a$-axis indicating the onset of space charge limited current (SCLC) in which carriers are trapped to sites in the lattice requiring a certain voltage to be completely filled. The space charge limited current density is proportional to the three halves power of the applied voltage along the $c$-axis.

As it is readable from Fig. 1a, the current density along the $a$-axis is greater than that along the $c$-axis by at least one order of magnitude. As for example, at a voltage of $2.0 \mathrm{~V}$, the current density is 1.35 and $20.75 \mathrm{~mA} / \mathrm{cm}^{2}$ for the $c$ - and $a$-axis measurements, respectively. The difference in these values is mainly due to the crystal anisotropy [9]. The value of the electrical anisotropy may be attributed to the high concentration of the stacking faults arising from weak inter-chain bonding [11] and/or the high anisotropy of the direction-dependent effective masses in the crystal [12].

Figure 1b represents the temperature dependence of resistivity being recorded in the temperature region 300 $400 \mathrm{~K}$ at constant electric field value in the ohmic region. The figure is a continuum of our previous work [9] in which the resistivity data was recorded in the temperature region of $110-320 \mathrm{~K}$. The low temperature electrical resistivity analysis revealed two energy levels located at 0.26 and $0.20 \mathrm{eV}$ along the $c$ - and $a$-axis, respectively [9]. Although the $\ln (\rho)-T^{-1}$ analysis, presented in Fig. 1b, reflected no change in the resistivity activation energy $(0.2 \mathrm{eV})$ along the $a$-axis, it exhibits a pronounced change from 0.26 to $0.41 \mathrm{eV}$ above $330 \mathrm{~K}$ along the $c$-axis. The value of resistivity activation energy being $0.41 \mathrm{eV}$ coincides with $E_{\mathrm{g}} / 2$ (middle of energy band gap) and results in an energy band gap of $0.82 \mathrm{eV}$ indicating that the crystal convert from extrinsic to intrinsic type semiconductor along the $c$-axis at temperature of $330 \mathrm{~K}$. The energy band gap of $\mathrm{TlGaTe}_{2}$ being $0.82 \mathrm{eV}$ is close to that determined from band structure analysis as $0.86 \mathrm{eV}$ [13] and as $0.84 \mathrm{eV}$ [14].

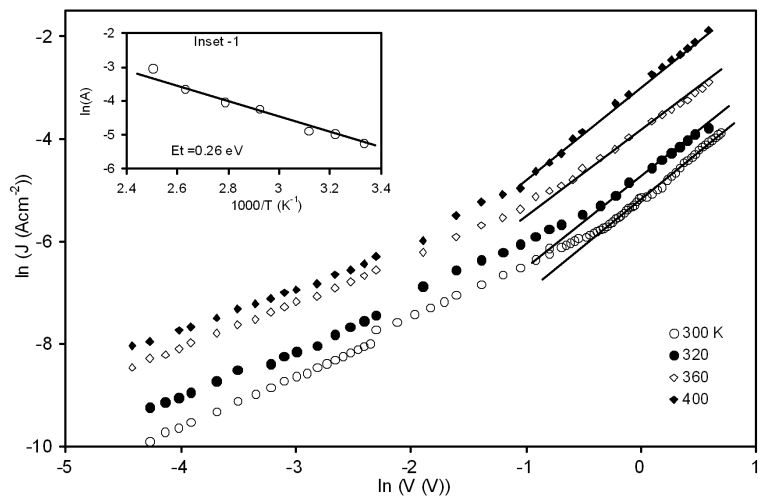

Fig. 2. The variation of current density versus applied voltage. The inset illustrates $\ln (A)-T^{-1}$ dependence.

Figure 2 displays the temperature dependence of the current density-voltage characteristics being recorded along the $a$-axis. The curves are very similar to that observed at room temperature. For single-carrier injection and discrete traps, the equation for the SCLC takes the form [15]:

$$
J=\frac{9}{8} \theta \mu \epsilon_{\mathrm{s}} \frac{V^{2}}{L^{3}},
$$

where $\epsilon_{\mathrm{S}}$ is the static dielectric constant of the material, $L$ is the distance between the electrodes, $\mu$ is the mobility and $\theta$ is the ratio of the free carrier density to the trapped carrier density. For hole injection and hole trapping, $\theta$ is given by 


$$
\theta=\frac{p}{p_{\mathrm{t}}}=\frac{g N_{\mathrm{v}}}{P_{\mathrm{t}}} \exp \left(-\frac{E_{\mathrm{t}}}{k T}\right) .
$$

Here, $P_{\mathrm{t}}$ is the trapped hole density (the crystals are of $p$-type conduction [9]), $N_{\mathrm{v}}$ is the effective density of states for the valence band, $g$ is the degeneracy factor and $E_{\mathrm{t}}$ is the energy of trap level. By inserting Eq. (2) into (1), the $J-V$ dependence becomes,

$$
J=\frac{9}{8} \mu \epsilon_{\mathrm{s}} \frac{g N_{\mathrm{v}}}{P_{\mathrm{t}}} \exp \left(-\frac{E_{\mathrm{t}}}{k T}\right) \frac{V^{2}}{L^{3}}=A V^{2},
$$

with

$$
A=\frac{9 \mu \epsilon_{\mathrm{s}}}{8 L^{3}} \frac{g N_{\mathrm{v}}}{P_{\mathrm{t}}} \exp \left(-\frac{E_{\mathrm{t}}}{k T}\right) .
$$

The $J-V$ plots (see Fig. 2) in the SCLC region at different temperatures were observed to correspond to discrete trapping levels because of the large quadratic region following the ohmic one. Using the intercepts $(\ln (A))$ of the $\ln (J)-\ln (V)$ variations in the quadratic regions, and plotting it as a function of reciprocal temperature as presented in inset 1 of Fig. 2, the trap energy level was determined as $0.26 \mathrm{eV}$. By substituting the values of the Hall mobility, the valence band effective density, the distance between electrodes, the static dielectric constant and the degeneracy factor as $\mu=105 \mathrm{~cm}^{2} \mathrm{~V}^{-1} \mathrm{~s}^{-1}$, $N_{\mathrm{v}}=4.83 \times 10^{15}\left(\mathrm{~m}^{*}=0.73 m_{0}\right)^{3 / 2} T^{-3 / 2}, L=0.20 \mathrm{~cm}$, $\epsilon_{\mathrm{s}}=14.8$ [16], $g=2$ which are reported in the previous works $[9,10]$, the hole trap density values were calculated at room temperature from Eq. (4) and found to be $5.6 \times 10^{10} \mathrm{~cm}^{-3}$. The existence of this trap level may be attributed to the vacancies of $\mathrm{Tl}, \mathrm{Ga}$ or Te atoms in the crystals. Similar behavior of $J-V$ characteristics at similar temperatures $(260-340 \mathrm{~K})$ with $E_{\mathrm{t}}=0.33 \mathrm{eV}$ and $N_{\mathrm{t}}=(1.4-2.2) \times 10^{13} \mathrm{~cm}^{-3}$ was also reported for $\mathrm{TlGaSe}_{2}$ layered crystals [17].

The value of the trap energy level being $0.26 \mathrm{eV}$ which was determined from the $J-V$ plots of the $a$-axis measurement coincides with that determined from (the ohmic region measurements) resistivity analysis along the $c$-axis [10]. In spite of the pronounced anisotropy of the crystal, the same energy levels appears to exist along both directions, it only differs in the temperature domination region.

Figure 3a reflects the variation of the current density with applied electric field $(\varepsilon)$ being registered along the $a$-axis at different temperatures. For low applied electric fields $(\varepsilon<0.5 \mathrm{~V} / \mathrm{cm})$, the current density-temperature dependence is very weak. As $\varepsilon$ increases the $J-T^{-1}$ variations become more pronounced. At high electric field values $(\varepsilon>5.0 \mathrm{~V} / \mathrm{cm})$ the $J-T^{-1}$ variations are parallel and systematic. The slopes of the $\ln (J)-T^{-1}$ plots shown by solid lines in Fig. 3a in accordance to the relation, $J=J_{0} \exp \left(-E_{\mathrm{a}} / k T\right)$, revealed an electric field dependent activation energies $\left(E_{\mathrm{a}}\right)$. The field dependence of activation energy is shown in Fig. 3b. As may be seen from the plot, the activation energy exhibited a value of $0.15 \mathrm{eV}$ at $0.5 \mathrm{~V} / \mathrm{cm}$ and it increases to 0.22 at $3.5 \mathrm{~V} / \mathrm{cm}$ and reaches 0.26 at $6.0 \mathrm{~V} / \mathrm{cm}$ (space charge limited re-
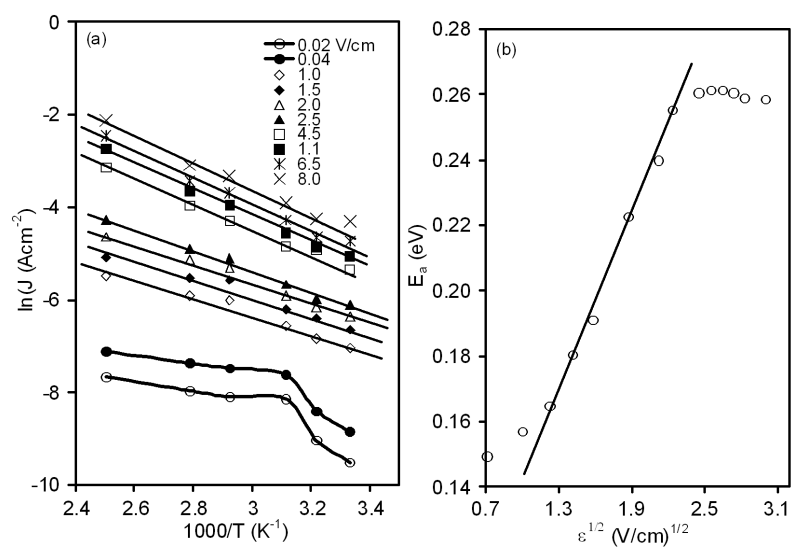

Fig. 3. (a) $\ln (J)-T^{-1}$ dependence along the $a$-axis. (b) The electric field dependence of activation energy along the $a$-axis.

gion) where then it remains constant. The constant value of $E_{\mathrm{a}}$ and its equality to $E_{\mathrm{t}}$ at high fields assures the discreteness of the trap sets in the space charge limited region.

The variation of the current density activation energy with electric field may be explained by means of the Poole-Frenkel effect [18] in which the current density takes the form,

$$
J \propto \varepsilon \exp \left(\frac{-q\left(\phi_{\mathrm{b}}-\sqrt{q \varepsilon / \pi \epsilon^{\prime}}\right)}{k T}\right) .
$$

Here, $\phi_{\mathrm{b}}$ is the voltage barrier (in zero applied electric field) that a hole must cross to move from one atom to another in the crystal, $\epsilon^{\prime}$ is the dynamic permittivity. In the Poole-Frenkel effect the holes are generally trapped in localized states. Occasionally, random thermal fluctuations will give the holes enough energy to get out of its localized state, and move to the valence band. Once there, the hole can move through the crystal, for a brief amount of time, before relaxing into another localized state. This effect describes how, in a large electric field, the hole does not need as much thermal energy to get into the valence band (since part of this energy comes from being pulled by the electric field), so it does not need as large a thermal fluctuation and will be able to move more frequently.

Figure 4 reflects the non-ohmic region of the $J-V$ characteristics being recorded at different temperatures along the $c$-axis. The solid lines which represent the linear slopes of the $\ln (J)-\ln (V)$ plots are parallel with slope values of approximately $3 / 2$. This type of behavior may be explained by the Child-Langmuir carrier transport law in semiconductors [19] in which the current density is given as

$$
J=J_{\mathrm{CL}}\left(\tau, n_{\mathrm{D}}\right) \sqrt{\frac{2 q}{m^{*}}} \epsilon_{\mathrm{s}} \frac{V^{3 / 2}}{L^{2}} .
$$

Here, $\epsilon_{\mathrm{S}}$ is the static dielectric constant of the material along the $c$-axis and $J_{\mathrm{CL}}\left(\tau, n_{\mathrm{D}}\right)$ is the Child-Langmuir 

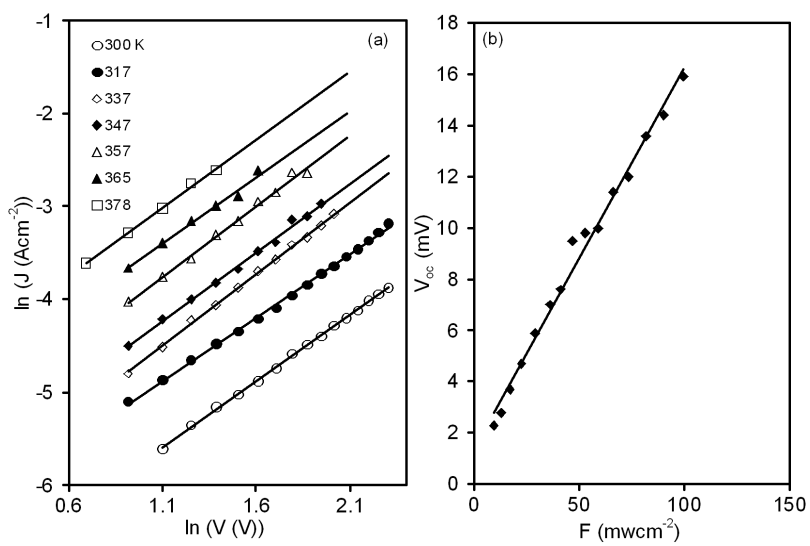

Fig. 4. (a) $\ln (J)-\ln (V)$ plots in the space charge region along the $c$-axis. (b) The illumination dependence of open circuit photovoltage.

current density which depends on carrier flight time $(\tau=$ $\sqrt{2 q / m^{*}} \xi V^{1 / 2} / L$ with $\xi$ being carrier relaxation time) and doping carrier density $\left(n_{\mathrm{D}}\right)$. The Child-Langmuir current density dominates when the applied potential between the electrodes is much larger than the thermal energy associated with the emitted carriers. In other words, when the current saturates at a maximal value determined by the condition that the electric field vanishes at the emission electrode (contact point) the crystal is said to operate under the Child-Langmuir space charge limited regime [19].

Figure $4 \mathrm{~b}$ displays the linear open-circuit photovoltage response $\left(V_{\text {oc }}\right)$ as a function of illumination intensity being recorded at zero bias voltage and at $300 \mathrm{~K}$, respectively. The data were recorded parallel to $c$-axis of the crystal. A systematic linear increase in $V_{\mathrm{oc}}$ is observed upon an increment in the light intensity $F$. The behavior is an indication of the photovoltaic property. The maximum photovoltage, obtained at an illumination intensity of $100 \mathrm{~mW} \mathrm{~cm}{ }^{-2}$ ( 0.70 s.un.), is equal to $16 \mathrm{mV}$. The corresponding short-circuit current density is $6.25 \mu \mathrm{A} \mathrm{cm}^{-2}$. These features of $\mathrm{TlGaTe}_{2}$ crystals are promising characteristics for using the crystals in solar cell fabrication if some modifications that improve the short circuit current density are done. The latter idea may be a purpose of future scientific research.

\section{Conclusions}

The anisotropic electrical and photovoltaic properties of $\mathrm{TlGaTe}_{2}$ crystals are investigated by means of current density-voltage characteristics in the temperature region of 300-400 K. The data analysis reflected a direction dependence of conduction. Namely, along the crystal's axis the current is governed by the Child-Langmuir current density which dominates when the applied potential between the electrodes is much larger than the thermal energy associated with the emitted carriers. The crystal along that axis exhibits intrinsic type of conduction.
Perpendicular to the crystal's axis, the crystal is extrinsic and the current density is limited by space charges which are trapped to sites in the lattice. For the same axis, the activation energy is found to be electric field dependent. This behavior is due to random thermal fluctuations will give the holes enough energy to get out of its localized state. $\mathrm{TlGaTe}_{2}$ crystals are observed to exhibit photovoltaic properties. The maximum open-circuit photovoltage and maximum short-circuit current density were found to be $16 \mathrm{mV}$ and $6.24 \mu \mathrm{A} \mathrm{cm}^{-2}$, respectively. These parameters are promising for the usage of these crystals as solar cells.

\section{References}

[1] D. Malterre, B. Dardel, M. Grioni, I. Weibel, Y. Baer, J. Phys. (France) IV 3, 97 (1993).

[2] R.M. Sardarli, O.A. Samedov, A.P. Abdullayev, E.K. Huseynov, F.T. Salmanov, G.R. Safarova, Semiconductors 44, 585 (2010).

[3] E.M. Gojaev, K.D. Gyul'mamedov, A.R. Ibragimova, A.A. Movsumov, Inorg. Mater. 46, 353 (2010).

[4] K. Mimura, T. Ishizu, K. Yamamoto, J. Takasu, Y. Yonehira, K. Wakita, N. Mamedov, Y. Taguchi, K. Ichikawa, K. Yan, E. Ikenaga, K. Kobayashi, Phys. Status Solidi C 6, 993 (2009).

[5] M.N. Nassary, S.A. Hussein, A.T. Nagat, Cryst. Res. Technol. 29, 869 (1994).

[6] M.P. Hanias, A. Anagnostopoulos, Phys. Rev. B 47, 4261 (1993).

[7] M.M. Kurbanov, Inorg. Mater. 41, 1277 (2005).

[8] K. Okazaki, K. Tanaka, J. Matsuno, A. Fujimori, L.F. Mattheiss, S. Iida, E. Kerimova, N. Mamedov, Phys. Rev. B 64, 045210 (2001).

[9] A.F. Qasrawi, N.M. Gasanly, J. Phys., Condens. Matter 21, 235802 (2009).

[10] A.F. Qasrawi, N.M. Gasanly, Phys. Status Solidi A 206, 2555 (2009).

[11] M. Parlak, C. Ercelebi, I. Gunal, H. Ozkan, N.M. Gasanly, Cryst. Res. Technol. 31, 673 (1996).

[12] V. Riede, H. Neumann, H. Sobotta, Solid State Commun. 38, 71 (1981).

[13] É.M. Godzhaev, G.S. Orudzhev, D.M. Kafarova, Phys. Solid State 46, 833 (2004).

[14] A.T. Nagat, G.A. Gamal, S.A. Hussein, Cryst. Res. Technol. 26, 19 (1991).

[15] M.A. Lampert, P. Mark, Current Injection in Solids, Academic Press, New York 1970.

[16] O. Madelung, Semiconductors: Data Handbook, 3rd ed., Springer, Berlin 2004.

[17] A.F. Qasrawi, N.M. Gasanly, Semicond. Sci. Technol. 19, 505 (2004).

[18] S.M. Sze, K.K. Ng, Physics of Semiconductor Devices, 2nd ed., Wiley, Hoboken, NJ 2007.

[19] N.B. Abdallah, P. Degond, A. Yamnahakki, Solid State Electron. 39, 737 (1996). 